\title{
Index
}

ACA, see Affordable Care Act

Affordable Care Act (ACA) 66-68, 70-73, 75-76, 78, 222

Anthropological Committee (Copenhagen) 96, 98-99

Armitage, David 22

art/artists 25, 140, 144-145, 150-153, 156-157, 197-198, 200, 212, 215-216, 219-222

fiction 187, 218, 222-223

graphic novel 73

opera 4,217

painting 152, 187, 220, 223

performance 4, 151, 216-217

poetry 218

Asilomar conference 9-95, 99

audience $2-9,12-13,186,220$

active audiences 114-115, 216

administrators as 185

anatomists' audiences 198-199

art lovers as 220

audience participation 142

audiences for controversial topics 5 , 89-94, 103-104, 162-163, 179, 222

citizens as 19, 109-110, 114-115, 122 consumers 109, 215

definition of 215-216, 223-224

depictions of 139

engaging audiences 114, 129

exhibitions and museums'

audiences 2, 141-143, 154-155,

195, 198, 200, 202, 205, 216,

221

historians as 185, 219

implicit/implied audience 5, 109, $165,170-172,178$

medical students as 24,198 , 218

physicians and health professionals as $8-9,43-44,52,54,61-62$, 109-110, 114, 120, 129-130, 198, 219-220

policy-makers and politicians as 3, 11-13, 36, 66-82, 129, 222

'produsers'/knowledge producers

as 4,223

public and $4-5,7,13,67,72,109$, $113-114,129-130$

audience research market research 215

media and cultural studies 3-7, 13, 19,215 
museum visitor studies 141-142,

215,222

reception studies $4,165,169,186$, $215-216$

audiencing 2-3, 5, 9, 13, 44, 89-90, 92-94, 104

definition of 2, 5, 90

Badgley, Robin 118-120, 123, 129

Barootes, Efstathios William (Staff) 115-116, 129

Baur, Nicole 139

Bengtsson, Bengt 169

Benjamin, Walter 184-186, 192, 202-203, 205

Beresford, Peter 141

Bergström-Walan, Maj-Briht 174-175

Berridge, Virginia 79

Bethlem Museum of the Mind see museum

bibliometrics 57-60, 74-75, 188

Bidloo, Govard 198-200, 203

Billings, John Shaw 188-190, 202

bioethics, see ethics

biomedicine 2, 10, 22-25, 29-30, $33-35,53-54,58,60$, 95, 101, 185,191

biotechnology 5, 89-91

Blease, Charlotte 10, 12

Bologna Declaration 20-21

Bondio, Mariacarla Gadebusch 45

Boris, Eileen 76

British Medical Journal 31, 116

Broberg, Gunnar 12

Bulletin for the History of Medicine 58

Bush, Vannevar 94

Butsch, Richard 114, 139

\section{Canadian Medical Association Journal} 117,120

Cate, Olle ten 23-24

citations (indexes), see bibliometrics citizen-physician 20, 22, 25, 28

citizenship 19-20, 31, 37, 110

Clinton, Bill 74, 78

cloning 89, 95, 99

compulsory sterilization, see sterilization

Cook, Harold 24

Cooter, Roger 193

co-production 99-101, 140-143, 149

as service user participation in mental health history research 138-157

Cullhed, Sture 174-176

Degler, Carl 80

de Lairesse, Gerard 198-199

democracy $7,18,22,30-32,101,112$, 140-141, 163, 205

Dinshaw, Carolyn 208

Donizetti, Gaetano 217

Donnelly, Kevin 78

Donoghue, Frank 57-58

Douglas, Tommy 107-108, 111-112, $115,117-119,121,125-128$

Duffin, Jacalyn 35

Eberwein, Robert 172

Edelman, Lee 210-211

Ehrlich, Paul 172

Elliot, Beth 148

Ellis, Rob 146

Entrustable professional activities (EPAs) 24-25

EPAs, see Entrustable professional activities

eugenics 5, 89-93, 96-104

ethics 2, 21, 23-26, 31, 34, 43-46, $48-54,56,62,80,89,91-92,95$, 102-104, 116, 222

European Research Council 51

exhibition 3, 9, 11, 138, 141-143, 145, 151, 185-186, 188, 191-192, 
195-200, 205-206, 213, 216, 221-223

Dream Anatomy, exhibition at National Library of Medicine 194-205, 216

expertise 1, 7-8, 11-12, 19, 32-33, 45, $52,58,99,139,142,147,153$, 156, 224

see also sex education films, history of medicine and

Fangerau, Heiner 45

Fee, Elizabeth 184, 195, 205-207

film 2-3, 201, 215, 218, 222-223 see also sex education films

FLSA, see United States Fair Labor Standards Act

Foucault, Michel 94, 175

Fraser, Nancy 114

genetics 5, 89-100, 102, 104

German Medical Licensure Act 43, 45-46, 53

Ginsburg, Ruth Bader 70

Golden, Janet 76

grand challenges 93, 95-97, 100-103

Gruber, Jonathan 73

GTE, see History, Theory, Ethics of Medicine

Guldi, Jo 22

Habermas, Jürgen 4

Habilitation 50-53, 59

Hall, Stuart 6

Hammarfelt, Björn 57, 60

Harvey, William 189, 219-220

Haskell, Thomas 80

health (care) policy 2, 9, 67-68, $72-73,81,92,215$

health law and 21, 25, 89, 97, 102

history and 107-130, 222

health humanities, see medical humanities
Hegeler, Inge 174-175

Hegeler, Sten 174

Henne, Carolyn 198-199

history

academic 153, 222

activism and 32, 77, 79, 184, 204

advocacy and 79

as collecting 184-186, 202-205, 213

commodification of $60-61$

heroic 59, 62-63, 170-172, 177, 193, 204, 224

historicity/historicism 77, 193, 208, 212

historiography $113,117-122$, 129-130

as idling/pleasure 193, 201-202, 204-205, 207, 213

personal 209-210

uses of 7-9, 26, 186-188, 192

History and Policy Network 222

History News Network 76

History, Theory, Ethics of Medicine

(Geschichte, Theorie, Ethik der

Medizin, GTE) 45-46, 49-55, $57-58,60$

Holocaust 89

Horizon 202093

Horton, Richard 22

Houston, C. J. 125-127

Houston, C. Stuart 124-128

HPV, see human papilloma virus

human papilloma virus (HPV) 29-33, 35

humanities 2, 6-11, 22, 44, 49, 51, 54-61, 67, 74, 81, 191, 205, 215, 221

impact 7-12, 33-35, 43, 58-61, 67, 75-76, 141, 154, 200

assessment/evaluation and 2, 7-12,

$74,81-82,186-188$

audiences and 3 
stakeholders and 7-8, 11 see also bibliometrics; media, impact and policy; publication/ publishing; research evaluation;

Research Excellence Framework in vitro fertilization (IVF) 89, 95, 99, 102

innovation 7-8, 21, 27, 98-99

Institute of human genetics and eugenics (Copenhagen) 92, 98-99

interdisciplinarity $2,25,44,53-54,56$, 61

Isis 58

IVF, see in vitro fertilization

Jasanoff, Sheila 99, 140

Johannsen, Wilhelm 96-97

Jost, Timothy 69-70

Journal of Policy History 78

Kelly, Arthur D. 116-118, 120-121, 127, 129

Keynes, Geoffrey 220

King v. Burwell 70-71

Kinsey, Alfred C. 175

Klein, Jennifer 76

Koselleck, Reinhart 93

Kristof, Nicholas 68

Kühl, Stefan 55-56

Kuhn, Annette 170-171

Lerner, Barron 76

Lévi-Strauss, Claude 213

Livingstone, Sonia 4-5, 109, 113

Lloyd, Woodrow 112, 115, 127

LOM-system (Leistungsorientierte Mittelvergabe) 55, 57-60

Long, Vicky 147

Lorenz, Chris 54-55

Lunt, Peter 5, 113

Lynch, Bernadette 142
MacTaggart, Ken 120-123, 127

Markel, Howard 76

Masters, William H. 175-176, 178

McLuhan, Marshall 6

media 113-114, 149, 162

artefacts/objects 146, 153, 184-185, 189, 192, 195-197, 199-203, 208-209, 215, 219

audiences and 3-7, 89-90

fiction 218

film 2-3, 189, 191-192, 201, 215, $218,222-223$

illustrations 191, 198-200

impact and 11, 67, 75-76

Internet 216

photographs 138, 189

print 44-45, 55, 58-59, 78, 111, 114, 116-120, 123-128, 164, 185-186, 188-189, 191, 198-200, 206-207, 209, 213, 218-219, 221-222

social media 9, 29-33, 66, 68, 75-77, 146, 150-151, 185-186, 188, 191, 201, 205, 207, 213, 217, 221-222

television 1-2, 32, 73-74, 107-108, $114-115,117,127,222$

see also exhibitions; museum; sex education films

Medicaid 66, 69-70, 75

Medicare (Canada) 4, 107-117, 122, 124-130

Medicare (US) 69-70, 75, 79

medical education/schools 2-3, 9, 11, 20-24, 43-46, 49, 51-52, 61, 74, $215,218-219$

mental health education 139 , 150

see also sex education films

medical humanities 2, 7-8, 10, 20, 24-26, 33-35, 37, 218, 221

medical liberalism 108, 111, 128 
mental health (care) 11-12, 90, 92, 96-97, 103, 138-57

Milliken, Christie 173

museum 2-3, 11, 48, 139, 141-144, 150-151, 154, 189, 203, 212, 215, 220, 222-223

art and 140, 144-146, 150-153, 156

Bethlem Museum of the Mind 11, 138, 144-146, 149-150, 153, $155-157$

National Federation of Independent Businesses v. Sebelius (NFIB v. Sebelius) 66, 69, 70-71

National Institutes of Health 185, 189, 191-192, 205

National Library of Medicine 185, 188-192, 195, 205-207

see also exhibition

Naylor, C. David 122-126, 128-129

nazism 62, 92, 95

New Public Management 7, 54-55, 62

New York Times 68, 72, 74-76, 200

New Yorker 75

NFIB v. Sebelius, see National Federation of Independent Businesses v. Sebelius

Nietzsche, Friedrich 193-194, 202, 205, 207

Northwood, Victoria 150

Nursing Clio (blog) 77

Nursing, history of $77,149,155$, $217-218$

Obama, Barack 66, 68, 72, 76-79 oral history 138-140, 145, 149, 154, 156

Osler, William 220

Ottesen-Jensen, Elise 164

Palin, Sarah 68

peer review 27, 58-59
Perspectives 66

Plos Biology 27

Plos Medicine 27

PNAS 27

Politifact 68

Polke, Sigmar 212

Portelli, Alessandro 139

Porter, Roy 36-37, 218, 221

Posner, Miriam 166

public engagement

in the history of mental health 138-142, 144-145, 149-152, 154-157

with science and technology 7 public history 154, 222-224

public sphere 4, 109, 113-114, 128, 130 public understanding of science 11 publications/publishing 55, 57-59, 61, 69, 73-74, 81, 114, 188, 195, 206-207, 220, 222

Quadagno, Jill 71

queer theory 210-212

racial hygiene/biology, see eugenics

Reeves, Max 143, 151-152

REF, see Research Excellence Framework

research assessment, see research evaluation

research evaluation $7-8,10,12,28$, $33-35,57,67,81-82,101$, 154-155

Research Excellence Framework (REF) 9-12, 81, 217

research funding 9-10, 16, 44, 54-59, 61-62, 93-94, 154

research policy 3, 6-7, 12-13, 93

Reverby, Susan 12, 72, 80, 204

Rochefort, David A. 78

Rockefeller Foundation 30, 98, 100

Rose, Nikolas 94 
Rosenberg, Charles E. 69

Rosner, David 204

Russell, David 139-140

Russo, Jasna 141

Schaefer, Eric 166, 171, 173

Science and Technology Studies (STS) 7, 13, 29, 99

Science in Transition 24, 27-28

science policy, see research policy

scientific literacy 25, 27-29, 32, 34

Sennett, Richard 4

sex education films 5-6, 162-183, 215

censorship and 6, 162-165, $167-169,173-174,176-179$

commercial cinema and 162-163, 165-166, 169-171, 173, 177-178 educational aspects of 165,167 , 170-174, 177, 179

history of medicine and 164-165, 167, 169-170, 174-178

Kärlekslivets offer ('Victims of Love') 171-173

Language of Love (Kärlekens språk) 174, 176-177

medicalization and 5, 164-165, 178 representation of physicians and scientists in 170-172, 177-178

Shapin, Steven 1

Small, Helen 22

Social History of Medicine 58

Sprengel, Kurt 19, 36-37

Starr, Paul 71, 74, 78

Steincke, Karl Kristian 97-98 sterilization 12, 90-92, 96-97, 99-103

stigma, reduction of 139,141 , 145-146, 150, 154, 157

Strohschneider, Peter 56

STS, see Science and Technology Studies

The Economist 28

The Lancet 22, 28, 116

The Triple Helix 27

Trump, Donald 68

Tydén, Mattias 12

UMC, see University Medical Center at Utrecht

United States Fair Labor Standards Act (FLSA) 76

United States Supreme Court 66-67, 69-71

University Medical Center at Utrecht (UMC) 11, 20, 23

vaccination see human papilloma virus (HPV)

Washington Post 75, 200

welfare state $90,98,108-109,127$, 178

Widerström, Karolina 1, 164

Williams, Linda 173

Wolfe, Sam 118-120, 123, 129

Zelizer, Julian 75

Zinn, Howard 80 